\title{
Vaccines and drugs under clinical trials for prevention and treatment of COVID-19
}

\author{
Umesh C. S. Yadav ${ }^{1}$
}

Received: 28 June 2020/ Accepted: 14 December 2020/Published online: 22 March 2021

(C) Indian Virological Society 2021

\begin{abstract}
The uncertainty related to prevention and treatment of Coronavirus disease 2019 due to lack of effective vaccine candidates or drug molecules has resulted in extensive spread of infection and mortality worldwide. Although the asymptomatic or mild patients are becoming healthy with regular over-the-counter medicines and proper rest and care, for the severe patients, in the absence of definite cure, different drug combinations are being used to treat on trial basis without the assurance of efficacy and safety. This scenario has however changed now with some medicines including antiviral Remdesivir and Favipiravir and anti-inflammatory drugs like dexamethasone and tocilizumab which have shown some positive results in trials such as decreasing need of mechanical or non-invasive ventilation or mortality. Further, a number of vaccine candidates are currently in pipeline and in advance stages of clinical trials, which will enhance their prospects in determining how the disease will be controlled in the times to come. In this article, an account of the under-trial potential drugs and vaccine candidates has been provided, and their future prospects have been discussed.
\end{abstract}

Keywords Coronavirus - COVID19 - Vaccines · Pandemic $\cdot$ Severe acute respiratory syndrome $\cdot$ SARS$\mathrm{CoV}-2$

Umesh C. S. Yadav

ucsyadav@mail.jnu.ac.in

1 Special Centre for Molecular Medicine, Jawaharlal Nehru University, New Delhi 110067, India

\section{Introduction}

The Coronavirus disease 2019 (COVID-19) has reached a pandemic proportion and as of November 7, 2020 has affected more than 49 million people and killed 1,239,157 people globally [17]. Amid the gradual easing of global lockdown, which was imposed in many countries in the first quarter of year 2020, the hospitals are struggling to keep up with the increased pace of patients arriving daily. Majority of the patients (nearly 80\%) are either asymptomatic or having mild to moderate symptoms including fever, dry cough, loss of sense of smell and taste and difficulty in breathing [16]. In many countries asymptomatic and mild patients are being advised to undergo in isolation and treated for the symptoms at home with normal medicines as used in case of seasonal flu. Such patients are getting recovered even without much attention from specialist doctors. They usually are advised to rest, stay hydrated, monitor their health regularly, and if needed over the counter antipyretics like acetaminophen/paracetamol to relive their fever is suggested [11]. To avoid the spread of infection to others, the asymptomatic and mild to moderate patients are further being advised to stay at home in isolation even from other family members and follow the precautionary measures that include wearing face masks, washing or sanitizing hands frequently, secure their cough or sneeze, keep safe distance from others and disinfect common places regularly.

Some moderate patients develop serious symptoms which includes increased difficulty in breathing, chest pain, delirium or confusion and lips and face getting bluish indicating lack of oxygen in blood [11]. Such patients need to be rushed to hospitals for additional care, support, and treatment. Once in the hospital, these patients are given fluids through intravenous tubes and oxygen through tubes 
inserted in nostrils and are tested for COVID-19 using PCR-based test and/or antigen-based test along with x-ray or CT scan of the chest [11].

In severe cases when patients fail to breath on their own, they are kept on ventilators to support their lungs and keep them oxygenated. As far as therapy of the serious patients is concerned there is no one specific therapeutic regimen. In fact, no specific medicine to treat COVID-19 at this time is available. Many candidate drugs are being used by doctors in different countries and hospitals, but their effects on COVID-19 and to treat the disease successfully have not been unequivocal.

An account of potential drug candidates and vaccines that are under trial or being repurposed to manage the COVID-19 patients has been presented here to understand their prospect of availability sooner or later to contain the pandemic in near future.

\section{Drug candidates against COVID-19}

Since the majority of serious COVID-19 patients are found to have other comorbid conditions such as obesity, diabetes, hypertension and other cardiopulmonary conditions, it is challenging to clinically intervene and save such patients, especially when the disease is presenting differently in different cohort of patients. Thus, the drugs that are being administered are mostly on the trial basis, which are currently small and single center-based studies, and the results are not highly unequivocal.

A small open-label non-randomized clinical trial was done initially in France where doctors used hydroxychloroquine, a drug usually used in malaria and rheumatoid arthritis, and azithromycin, which is an antibiotic, to study their impact on COVID-19. The results claimed that hydroxychloroquine decreased the viral load in COVID-19 patients six days after the treatment, and that its effect was strengthened by adding azithromycin to the treatment regimen [21]. This study was small where 20 patients were included in single arm protocol.

The American Food and Drug Administration (FDA), based on the results of above study, gave approval for the emergency use of antimalarial drugs hydroxychloroquine and chloroquine to treat COVID-19 patients but cautioned against use outside of the hospital setting or a clinical trial due to risk of heart rhythm problems [24]. The effects of these antimalarial drug are however controversial. Another study showed no benefit from hydroxychloroquine treatment, rather increased likeliness of patients to die. In this study, which was conducted on 96,032 COVID-19 patients, the death in control group (who did not receive the drug) was only $9.3 \%$, while in the group treated with hydroxychloroquine death was reported in $18 \%$ of the patients and those treated with chloroquine $16.4 \%$ death was reported. Further, when these drugs were combined with an antibiotic, in place of benefiting, the death rate increased to 23.8 and $22.2 \%$, respectively [29]. Although these drugs are otherwise safe when used for malaria or rheumatoid arthritis, but in the COVID-19 patients with additional comorbid conditions like metabolic disorders, heart problems, kidney disease, diabetes, asthma etc. it is likely to enhance the complications exhibiting side effects such as arrythmia, muscle loss, seizures, and decreased blood cell count [29]. With these results, while the FDA has now cautioned the used of both the drugs in COVID-19 patients, a group of more than hundred scientists from across the world raised the questions on the statistical accuracy and data validation and the above study stands retracted [38].

The US FDA also gave an emergency use authorization for the use of another drug Remdesivir [15]. It is a nucleotide analogue which prevents viral replication by inhibiting RNA dependent RNA polymerase enzyme in cell-based assays, and thus can be helpful in viral diseases [22]. However, its effect in COVID-19 patients was not very strong. At best, in the adult COVID-19 patients hospitalized with lower respiratory tract infection Remdesivir was found superior to placebo in decreasing the recovery time [8]. According to the FDA, Remdisivir was allowed for treatment of severe COVID-19 patients with low oxygen level requiring ventilators, and its use would be considered experimental. Similarly, Favipiravir, another antiviral drug, has also been found effective against the mild to moderate COVID-19 conditions and has been approved by many countries for restricted use. In a metaanalysis study Favipiravir showed significant clinical and radiological improvement compared to the standard care although no significant differences on viral clearance, oxygen support requirement and side effect profiles were observed [39]. Although antiviral drugs were seen as potential treatment strategy, trails with these drugs have shown satisfactory and beneficial effects against COVID19. More studies and clinical trials are underway to explore the possibility of developing new antivirals or allow the use of present drugs through repurposing them.

Interleukin (IL)-6 neutralizing antibody, tocilizumab, has also emerged as an alternative drug to treat COVID-19. Tocilizumab is usually used to treat autoimmune conditions where the level of cytokine IL- 6 is reportedly elevated. A single centre study done in 15 COVID-19 patients at Zhongfaxincheng campus of Tongji Hospital in Wuhan, China, suggested that drug tocilizumab can be an effective treatment option in COVID-19 patients with elevated IL-6 having a risk of 'cytokine storm' [27]. According to an observational study recently published in the Lancet rheumatology, in the COVID-19 patients needing intensive care unit admission, tocilizumab helped decrease mortality 
although the results from randomized trial is still awaited [10]. In another randomized clinical trial, it was found that tocilizumab could lower the initial requirement of noninvasive ventilation or death by 14 th day but did not decrease mortality by 28th day [23]. The CORIMUNO19-TOCI trail in France undertook a multicenter openlabel randomized controlled study of tocilizumab involving adult patients with moderate or severe COVID19 not needing intensive care support. The study set the need for non-invasive/mechanical ventilation or death at day 14 after admission as primary outcome. Out of 129 patients who participated in the study, 65 were given standard care along with tocilizumab and the other 64 were given only standard care. In a press release, the study reported preliminarily that a significantly lower proportion of patients in tocilizumab group required ventilator support or died at day 14 than the standard care group [42]. Another retrospective observational study performed in small group of COVID-19 patients reported effectiveness of tocilizumab in critically ill COVID-19 patients and recommended that repeated doses of tocilizumab could be an effective treatment option especially in patients with a risk of cytokine storm [28]. Despite the positive outcomes reported in these studies, more recent studies have raised caution due to variance in different cohort of patients. For example, a study by Stone et al. reported that "tocilizumab was not effective for preventing intubation or death in moderately ill hospitalized patients with Covid-19." Although the authors did not rule out 'some benefit or harm' due to wide confidence intervals for efficacy comparisons in the study [41]. In view of the mixed results, experts have asked to reassess the role of this drug in the COVID19 patients and agencies like National Institute of Health (NIH), USA recommended against its use except only in the context of a clinical trial [33].

Cytokines are the chemicals secreted by the immune cells in response to the invading pathogens with the aim to neutralize and remove them from the body. However, in case of high pathogen load and sever infection these cells secrete overwhelmingly large amounts of cytokines, which is referred to as 'cytokine storm', leading to the damage of host tissue and organs. Involvement of NALP3 (NACHT, LRR, and PYD domains-containing protein 3) inflammasome, an important component of innate immune arm of the body, in the virus mediated inflammation is known and it is likely to have role in the 'cytokine storm' during COVID-19 as well [45, 46]. The viral proteins and RNA are known to activate NALP3 inflammasome in the macrophages through pathogen associated molecular pattern (PAMP) and danger associated molecular pattern (DAMP) recognition receptors and produce excessive amount of proinflammatory cytokines IL-1 $\beta$ [26]. This and other inflammatory cytokines such as IL- 6 and TNF- $\alpha$ released by immune cells can cause cytokine storm leading to severe pulmonary disease upon novel Coronavirus infection. The swift and strong immune response lodged by NALP3 inflammasome, which opens cytokines floodgates, results in aggravation of the disease severity and increases fatality, making it an important molecular target to treat COVID-19 [20, 44]. Thus, in the severe COVID-19 patients, to contain the cytokine storm and to enhance the treatment efficiency use of cytokine neutralizing antibodies or other potent anti-inflammatory molecules can be effective in decreasing the inflammation. There can be many targets associated with NALP3 pathway including NALP3 itself, and IL-1 $\beta$. The drug molecules are already available against these mediators and are in different stages of clinical trials which can be repurposed for the treatment of COVID-19 patients. For example, tranilast for NALP3 inhibition has been used in bronchial asthma and suggested for repurposing for the treatment of COVID-19 [9]. Similarly, an anti-IL-1 $\beta$ monoclonal antibody canakinumab and human IL-1 receptor antagonist anakinra can be good candidates for the inhibition of bioactive IL-1 $\beta$ and suppression of cytokine storm in COVID-19 patients [9, 35].

It should be understood that virus per se does not cause the severity of the disease, rather it's the heightened inflammation lodged by our immune cells in response to the virus infection that affect the function of cells and tissues. Thus, decreasing the level of inflammation helps patients minimize the collateral damage and buy the time for the immune cells to fight the infection. Accordingly, other potent anti-inflammatory drugs and monoclonal antibodies against cytokines like IL- 8 and TNF- $\alpha$ can also be tested. Since inflammation during viral infection is common and known to cause collateral damage by killing the host cells and weakening the response against the virus, this can be effective strategy against COVID-19.

The clinical trials using the plasma-derived from the blood of recovered patients have been done with mixed results. Convalescent Plasma, as it is called, contains the antibodies generated against the Coronavirus in the recovered patient, and is used to kill the virus in other patients [43]. Various agencies have allowed its use in severely ill COVID-19 patients and for the purpose of clinical trials including the FDA, which authorized the emergency use of antibody-packed plasma isolated from recovered COVID-19 patients [36]. A recently published results from an open label phase II multicenter randomized controlled trial (PLACID Trial) in India funded by the ICMR suggested outrightly that therapy with convalescent plasma did not show "a reduction in progression to severe covid-19 or all cause mortality" [4]. However, the authors also suggested that "A priori measurement of neutralizing antibody titers in donors and participants might further 
clarify the role of convalescent plasma in the management of covid-19." [4].

In addition to these potential drug candidates which are in different stages of trials or being used in severe patients as emergency medicines, WHO had launched a SOLIDARITY trail as an international clinical study for COVID-19 treatment and enrolled nearly 12,000 patients in over 30 countries selecting 500 hospitals [40]. The Solidarity trial aimed to compare its drugs versus standard of care and assessed their relative effectiveness against COVID-19 on three major outcomes i.e. mortality, need for assisted ventilation and duration of hospital stay. According to the last update released on 15 October 2020, the trial reported that its four treatment options including Remdesivir, hydroxychloroquine, lopinavir/ritonavir and interferon showed 'little or no effect on overall mortality, need of ventilation and duration of hospital stay' in COVID-19 patients admitted in hospitals [40]. However, trial suggested that only corticosteroids were found effective against severe and critical cases of COVID-19 [40].

\section{Vaccine candidates against COVID-19}

Several vaccine candidates are also under the pipeline across the world which are aimed to be used as preventive tool against Coronavirus infection. Vaccines are substances derived from disease causing organisms that when enter human body induce acquired immunity against disease causing organisms. A vaccine is usually made by weakening the pathogen by a process called attenuation, or is a heat-killed pathogen, or its disease-causing toxins or proteins present on their outer surface.

The vaccines against a virus can be of different types depending upon the technological platforms used to synthesize them. These may include inactivated or live attenuated viral particles, virus-like particles not containing viral genome, recombinant viral proteins or peptides, viral nucleic acids encapsulated in delivery vesicles like liposomes or inserted in plasmid vector, and viral vectors which may or may not replicate in host cells. Globally, several efforts are being made to make the vaccines in each of these different platforms. According to an estimate, there are more than hundred candidate vaccines that are currently in the developmental pipeline and at different stages of clinical trials, some having advanced to phase $3 / 4$, while a couple of them have been approved recently in many countries for vaccination of adults aged 18 years or above.

In one of the initial human trials, a COVID-19 vaccine was developed using an adenovirus type 5 (common cold virus) vector containing partial genetic material of Coronavirus that encodes the spike protein. Just one dose of this vaccine, named $\mathrm{Ad} 5-\mathrm{nCoV}$, found to generate specific T-cells and antibodies against the Coronavirus and was also found safe [47]. The study, which as an open-label phase 1 clinical trial performed on 108 healthy volunteers from Wuhan, China, demonstrated encouraging outcomes. In this trial, the vaccine induced rapid specific $\mathrm{T}$-cell responses on day 14 post-vaccination and was found to be tolerable and immunogenic even 28 days after the injection of the vaccine [47]. The group working on this candidate vaccine, CanSino Biologics of China, has already completed phase 2 study and found that Ad5-nCoV vaccine was safe and after a single immunization dose it induced significant immune responses in the majority of recipients [48]. The vaccine is currently under phase 3 trial (ClinicalTrials.gov Identifier: NCT04526990) to evaluate efficacy, safety and immunogenicity of the candidate vaccine [34].

Another candidate vaccine in trial pipeline is a nucleic acid vaccine being developed by USA-based pharma company Moderna. They have used viral mRNA that codes for spike protein encapsulated into a liposome for phase 1 study (ClinicalTrials.gov number, NCT04283461). The company has claimed that their candidate vaccine, mRNA1273 was found safe and successfully induced immune response in the volunteers which was similar to that of natural viral infection [30]. The phase 1 study involved merely eight elder volunteers who showed only mild to moderate adverse events linked to mRNA-1273. The study suggested to use $100 \mu \mathrm{g}$ dose for phase 3 trial as this dose induced higher binding- and neutralizing-antibody titers which were better than that of a lower $25 \mu \mathrm{g}$ dose [6]. The company has enumerated on its website several advantages of mRNA-based vaccine such as similarity to natural viral infection, possibility of including several mRNAs coding for different viral proteins in a single vaccine. The candidate vaccine has entered phase 3 trial (ClinicalTrials.gov Identifier: NCT04470427) in the USA [3]. Recently, this vaccine candidate has been reported to show $94.1 \%$ efficacy in phase 3 trial and the makers have sought approval for distribution and use in adults of 18 years of age and above in the USA under emergency use authorization.

A Chinese company, SinoVac Biotech, announced that its inactivated vaccine candidate produced neutralizing antibodies against Coronavirus in the preclinical model including rhesus macaque monkeys and was found safe [14]. The company has already started phase 3 clinical trial using their Adsorbed COVID-19 candidate vaccine and more than 13 thousand volunteers [13]. Another company, China national pharmaceutical Sinopharm, has started phase 3 clinical trial using inactivated viral particles as vaccine, the first in this category to reach phase 3 stage [12]. Earlier, the company had claimed that in phase 1 trial, which involved injecting the vaccine in 96 healthy people, 
the vaccine returned no severe adverse reactions and showed good safety profile [18].

A DNA vaccine for COVID-19 named INO-4800 has been developed by Inovio Pharmaceuticals, Inc., a USbased company. Announcing the results of its pre-clinical study involving mice and guinea pigs, the company has claimed that the vaccine elicited 'robust neutralizing antibody and $\mathrm{T}$-cell immune responses against Coronavirus SARS-CoV-2' [25]. Phase 2 clinical trial (ClinicalTrials.gov Identifier: NCT04447781) of its candidate vaccine is underway in the USA [37].

A vaccine candidate named ChAdOx $1 \mathrm{nCoV}-19$, which uses a weakened common cold chimpanzee virus ChAdOx 1 containing the genetic material of the spike protein of Coronavirus has been developed by Oxford University [31]. Once the vaccine injected, it produces spike protein in host body which alerts the host immune system to produce immune response and produce antibodies against it. In a preliminary report of a phase $1 / 2$, single-blind, randomized controlled trial the candidate vaccine showed an acceptable safety, and increased antibody responses was observed upon homologous boosting [19]. The phase 3 clinical trial of ChAdOx $1 \mathrm{nCoV}-19$ vaccine (ClinicalTrials.gov Identifier: NCT04536051) has already begun [2]. As reported recently Oxford-Astrazeneca vaccine showed 90\% efficacy when half dose of the vaccine was followed up by a full dose after a month compared to $62 \%$ efficacy when two full dose of vaccine was administered a month apart.

In India also clinical trials are underway with at least three candidate vaccines. Indian Council of Medical Research (ICMR)-National Institute of Virology has collaborated with an indigenous biotech company Bharat Biotech International Ltd (BBIL) to develop a COVID-19 vaccine, named COVAXIN, which is whole-virion inactivated SARS-CoV-2 vaccine. A randomized, double-blind, and multicenter phase $1 / 2$ study using this candidate, code named BBV152, is currently underway to evaluate its safety, reactogenicity, tolerability and immunogenicity in healthy volunteers [5]. The BBIL has stated that its COVID-19 vaccine will be in the form of nasal-drop and named 'CoroFlu', which is built on an existing flu vaccine platform that has been found safe in humans. Another vaccine candidate under trail in India is Covishield which is under joint study in a Phase $2 / 3$, observer-blind, randomized trial being done by the Serum Institute of India (SII) and ICMR to examine safety and immunogenicity [1]. The pharma company Zydus Cadila of India has developed a plasmid DNA vaccine, named ZyCoV-D, for the prevention of COVID-19, which has been found safe in Phase I clinical trial done in healthy subjects. The company has begun a multisite, prospective, randomized, adaptive, phase 2 trial with an aim to evaluate the safety and immunogenicity of its vaccine candidate [7].
The vaccine development involves huge risks for the developers and manufacturers. The input cost is involved at different levels of development and production such as clinical trials, approval of the regulating bodies and compliance to their regulations. In addition, building manufacturing units for large-scale production, recruiting the human resources such as skilled labor and arranging required raw materials involve huge capital investment without surety of success. Further, vaccine development is a time taking enterprise and the window to develop one successfully is a minimum of 1.5 to 2 years, which increases the input cost even more. In the course of an ongoing pandemic, to develop an effective vaccine is challenging task and these challenges and pressures put paramount strain on the rapid production process.

\section{Future prospect}

Humanity is facing unprecedented health challenge due to COVID-19 pandemic. In the absence of clear therapeutic and preventive measures, the number of infected people and deaths due to infection both are soaring. Even though countries have eased the lockdown to allow the resumption of economic activities, WHO has warned that second wave could be more severe and may result in greater peak of infection and mortality. Indeed, in many countries the COVID-19 cases are consistently rising and in some other countries including Europe 2nd wave of infection has commenced and countries are resorting to lockdown again [32]. In these scenarios, it can be hoped that drugs and vaccines that are currently in pipeline as discussed above will be available sooner and will help contain the disease, reduce the severity and minimize the mortality. There are several challenges in transferring the undertrial drugs and vaccines to the clinics, but despite these challenges, the humanity is hopeful of having much needed breakthrough against the COVID-19 earlier than later.

\section{Compliance with ethical standards}

Conflict of interest The author declare that they have no conflict of interest.

\section{References}

1. A Phase 2/3. Observer-blind, randomized, controlled study to determine the safety and immunogenicity of covishield (COVID19 Vaccine) in healthy Indian adults. 7 Nov 2020. http://ctri.nic. in/Clinicaltrials/pmaindet2.php?trialid=46186\&EncHid=\&user Name $=$ CTRI/2020/08/027170.

2. A Study of a Candidate COVID-19 Vaccine (COV003). 7 Nov 2020. https://clinicaltrials.gov/ct2/show/NCT04536051. 
3. A Study to Evaluate. Efficacy, Safety, and Immunogenicity of mRNA-1273 vaccine in adults aged 18 years and older to prevent COVID-19. 7 Nov 2020. https://clinicaltrials.gov/ct2/show/ NCT04470427.

4. Agarwal A, Mukherjee A, Kumar G. Convalescent plasma in the management of moderate covid-19 in adults in India: open label phase II multicentre randomised controlled trial (PLACID trial). BMJ. 2020;371:m3939. https://doi.org/10.1136/bmj.m3939.

5. An Adaptive. Seamless phase 1, followed by phase 2 randomized, double-blind, multicenter study to evaluate the safety, reactogenicity, tolerability and immunogenicity of the whole-virion inactivated SARS-CoV-2 Vaccine (BBV152) in Healthy Volunteers. 7 Nov 2020. http://ctri.nic.in/Clinicaltrials/showallp. php mid1 $=45184 \&$ EncHid=\&userName=BBV152.

6. Anderson EJ, Rouphael NG, Widge AT, et al. Safety and immunogenicity of SARS-CoV-2 mRNA-1273 vaccine in older adults. N Engl J Med. 2020. https://doi.org/10.1056/ NEJMoa2028436.

7. A prospective, randomized, adaptive, phase I/II clinical study to evaluate the safety and immunogenicity of Novel Corona Virus2019-nCov vaccine candidate of M/s Cadila Healthcare Limited by intradermal route in healthy subjects. 8 Nov 2020. http://ctri. nic.in/Clinicaltrials/pmaindet2.php?trialid $=45306 \&$ EncHid $=$ \&userName=CTRI/2020/07/026352.

8. Beigel JH, Tomashek KM, Dodd LE, et al. Remdesivir for the treatment of Covid-19-final report. N Engl J Med. 2020. https:// doi.org/10.1056/NEJMoa2007764.

9. Bertocchi I, Foglietta F, Collotta D, et al. The hidden role of NLRP3 inflammasome in obesity-related COVID-19 exacerbations: Lessons for drug repurposing. $\mathrm{Br} \mathrm{J}$ Pharmacol. 2020;177:4921-30. https://doi.org/10.1111/bph.15229.

10. Biran N, Ip A, Ahn J, Go RC. Tocilizumab among patients with COVID-19 in the intensive care unit: a multicentre observational study. Lancet Rheumatol. 2020;2(10):e603-12. https://doi.org/10. 1016/S2665-9913(20)30277-0.

11. Centers for Disease Control and Prevention. 26 May 2020. https://www.cdc.gov/Coronavirus/2019-ncov/hcp/clinical-gui dance-management-patients.html.

12. China's Sinopharm launches. Phase III trial of Covid-19 vaccine in UAE. 7 Nov 2020. https://www.clinicaltrialsarena.com/news/ sinopharm-covid-vaccine-uae-trial/.

13. Clinical Trial of Efficacy and Safety of Sinovac's Adsorbed COVID-19 (Inactivated) Vaccine in Healthcare Professionals (PROFISCOV). 7 Nov 2020. https://clinicaltrials.gov/ct2/show/ NCT04456595.

14. Cohen J. COVID-19 vaccine protects monkeys from new Coronavirus, Chinese biotech reports. 26 May 2020. https://www. sciencemag.org/news/2020/04/covid-19-vaccine-protects-mon keys-new-Coronavirus-chinese-biotech-reports.

15. Coronavirus (COVID-19). Update: FDA issues emergency use authorization for potential COVID-19 treatment. 26 May 2020. https://www.fda.gov/news-events/press-announcements/Cor onavirus-covid-19-update-fda-issues-emergency-use-authoriza tion-potential-covid-19-treatment.

16. Coronavirus disease. 2019 (COVID-19) Situation report-46. World Health Organization; 2020. p. 2.

17. Coronavirus disease (COVID-19) pandemic. World Health Organization. 7 Nov 2020. https://www.who.int/emergencies/dis eases/novel-coronavirus-2019.

18. First inactivated COVID-19 vaccine enters 2 nd phase of clinical trial. 25 May 2020. https://news.cgtn.com/news/2020-04-25/ First-inactivated-COVID-19-vaccine-enters-2nd-phase-of-clin ical-trial-PZ1hJMIjhm/index.html.

19. Folegatti PM, Ewer KJ, Aley PK. Safety and immunogenicity of the ChAdOx1 nCoV-19 vaccine against SARS-CoV-2: a preliminary report of a phase $1 / 2$, single-blind, randomised controlled trial. Lancet. 2020;396(10249):467-78. https://doi.org/ 10.1016/S0140-6736(20)31604-4.

20. Freeman TL, Swartz TH. Targeting the NLRP3 inflammasome in severe COVID-19. Front Immunol. 2020;11:1518. https://doi.org/ 10.3389/fimmu.2020.01518.

21. Gautret P, Lagier JC, Parola P, et al. Hydroxychloroquine and azithromycin as a treatment of COVID-19: results of an openlabel non-randomized clinical trial. Int J Antimicrob Agents. 2020. https://doi.org/10.1016/j.ijantimicag.2020.105949.

22. Gordon CJ, Tchesnokov EP, Feng JY, Porter DP, Götte M. The antiviral compound remdesivir potently inhibits RNA-dependent RNA polymerase from Middle East respiratory syndrome Coronavirus. J Biol Chem. 2020;295(15):4773-9. https://doi.org/ 10.1074/jbc.AC120.013056.

23. Hermine O, Mariette X, Tharaux P, et al. Effect of Tocilizumab vs usual care in adults hospitalized with COVID-19 and moderate or severe pneumonia: a randomized clinical trial. JAMA Intern Med. 2020. https://doi.org/10.1001/jamainternmed.2020.6820.

24. Hydroxychloroquine or Chloroquine for COVID-19. Drug Safety Communication-FDA cautions against use outside of the hospital setting or a clinical trial due to risk of heart rhythm problems. 26 May 2020. https://www.fda.gov/drugs/drug-safety-andavailability/fda-cautions-against-use-hydroxychloroquine-orchloroquine-covid-19-outside-hospital-setting-or.

25. INOVIO's COVID-19 DNA Vaccine INO-4800. Demonstrates robust neutralizing antibody and $\mathrm{T}$ cell immune responses in preclinical models. 25 May 2020. http://ir.inovio.com/newsreleases/news-releases-details/2020/INOVIOs-COVID-19-DNAVaccine-INO-4800-Demonstrates-Robust-Neutralizing-Anti body-and-T-Cell-Immune-Responses-in-Preclinical-Models/ default.aspx.

26. Leavy O. Inflammasomes: polymeric assembly. Nat Rev Immunol. 2014;14(5):287. https://doi.org/10.1038/nri3669.

27. Luo P, Liu Y, Qiu L, Liu X, Liu D, Li J. Tocilizumab treatment in COVID-19: a single center experience. J Med Virol. 2020. https://doi.org/10.1002/jmv.25801.

28. Luo P, Liu Y, Qiu L, Liu X, Liu D, Li J. Tocilizumab treatment in COVID-19: a single center experience. J Med Virol. 2020;92(7):814-8. https://doi.org/10.1002/jmv.25801.

29. Mehra MR, Desai SS, Ruschitzka F, Patel AN. Hydroxychloroquine or chloroquine with or without a macrolide for treatment of COVID-19: a multinational registry analysis. Lancet. 2020;S0140-6736(20):31180-6. https://doi.org/10.1016/S01406736(20)31180-6.

30. Moderna announcement. 26 May 2020. https://investors.moder natx.com/news-releases/news-release-details/moderna-announ ces-positive-interim-phase-1-data-its-mrna-vaccine.

31. Oxford vaccine Group. COVID-19 vaccine development. 25 May $2020 . \quad$ https://www.ovg.ox.ac.uk/news/covid-19-vaccinedevelopment.

32. Page ML. Europe's second wave of coronavirus is starting to eclipse the first. 3 Nov 2020. https://www.newscientist.com/arti cle/2258904-europes-second-wave-of-coronavirus-is-starting-toeclipse-the-first/\#ixzz6dBwhknJ4.

33. Parr JB. Time to reassess Tocilizumab's role in COVID-19 pneumonia. JAMA Intern Med. 2020. https://doi.org/10.1001/ jamainternmed.2020.6557.

34. Phase III. Trial of A COVID-19 vaccine of adenovirus vector in adults 18 years old and above. 7 Nov 2020. https://clinicaltrials. gov/ct2/show/NCT04526990.

35. Pontali E, Volpi S, Antonucci G, et al. Safety and efficacy of early high-dose IV Anakinra in severe COVID-19 lung disease. J Allergy Clin Immunol. 2020;146(1):213-5. https://doi.org/10. 1016/j.jaci.2020.05.002.

36. Recommendations for Investigational COVID-19 convalescent plasma. 2 Sept 2020. https://www.fda.gov/vaccines-blood- 
biologics/investigational-new-drug-ind-or-device-exemption-ideprocess-cber/recommendations-investigational-covid-19-con valescent-plasma.

37. Safety. Tolerability and Immunogenicity of INO-4800 Followed by Electroporation in Healthy Volunteers for COVID19. 7 Nov 2020. https://clinicaltrials.gov/ct2/show/NCT04447781.

38. Scientists Question Validity of Major Hydroxychloroquine Study. 29 May 2020. https://www.nytimes.com/2020/05/29/health/Cor onavirus-hydroxychloroquine.html.

39. Shrestha DB, Budhathoki P, Khadka S, et al. Favipiravir versus other antiviral or standard of care for COVID-19 treatment: a rapid systematic review and meta-analysis. Virol J. 2020;17:141. https://doi.org/10.1186/s12985-020-01412-z.

40. "Solidarity" clinical trial for COVID-19 treatments. 7 Nov 2020. https://www.who.int/emergencies/diseases/novel-coronavirus2019/global-research-on-novel-coronavirus-2019-ncov/solidar ity-clinical-trial-for-covid-19-treatments.

41. Stone JH, Frigault MJ, Serling-Boyd NJ. Efficacy of Tocilizumab in patients hospitalized with Covid-19. N Engl J Med. 2020. https://doi.org/10.1056/NEJMoa2028836.

42. Tocilizumab improves significantly clinical outcomes of patients with moderate or severe COVID-19 pneumonia. 7 Nov 2020. https://pipelinereview.com/index.php/2020042874458/Anti bodies/Tocilizumab-improves-significantly-clinical-outcomes-ofpatients-with-moderate-or-severe-COVID-19-pneumonia.html.

43. UK researchers launch trial of blood plasma therapy for Covid19. 26 May 2020. https://www.clinicaltrialsarena.com/news/ukblood-plasma-covid-19-trial/.
44. Yap JKY, Moriyama M, Iwasaki A. Inflammasomes and pyroptosis as therapeutic targets for COVID-19. J Immunol. 2020;205:307-12. https://doi.org/10.4049/jimmunol.2000513.

45. Yu J, Wu Y, Wang J. Activation and role of NACHT, LRR, and PYD domains-containing protein 3 inflammasome in RNA viral infection. Front Immunol. 2017;8:1420. https://doi.org/10.3389/ fimmu.2017.01420.

46. Zhao C, Zhao W. NLRP3 inflammasome-a key player in antiviral responses. Front Immunol. 2020;11:211. https://doi.org/ 10.3389/fimmu.2020.00211.

47. Zhu FC, Li YH, Guan XH. Safety, tolerability, and immunogenicity of a recombinant adenovirus type-5 vectored COVID-19 vaccine: a dose-escalation, open-label, non-randomised, first-inhuman trial. Lancet. 2020;S0140-6736(20):31208-3. https://doi. org/10.1016/S0140-6736(20)31208-3.

48. Zhu FC, Guan XH, Li YH. Immunogenicity and safety of a recombinant adenovirus type-5-vectored COVID-19 vaccine in healthy adults aged 18 years or older: a randomised, double-blind, placebo-controlled, phase 2 trial. Lancet. 2020;396(10249):479-88. $\quad$ https://doi.org/10.1016/S01406736(20)31605-6.

Publisher's Note Springer Nature remains neutral with regard to jurisdictional claims in published maps and institutional affiliations. 